\section{GP137 A COMPARATIVE ANALYSIS OF CHILDREN AGED 4-13 YEARS WITH TYPE I DIABETES WHO WERE INVITED TO TAKE PART IN THE 'TEAM TYPE I' INITIATIVE}

${ }^{1}$ Kerrie Hennigan*, 1,2Maaz Mirza, 'Orla Neylon, ${ }^{3}$ Clodagh O'Gorman. ${ }^{1} U H L$, Limerick, Ireland; ${ }^{2}$ Graduate Entry Medical School, Limerick, Ireland; ${ }^{3}$ UHL, Limerick, Ireland

10.1136/archdischild-2019-epa.201

Aims To bring together a group of children with Type I Diabetes Mellitus (T1DM), with their siblings and parents, to participate in a group fun run. Alleviate any barriers to exercise they may previously have had by setting up a hypoglycaemia treatment table and having the option of chaperones to run with the children. Gather information about diabetes care and exercise by means of survey.

Methods Flyers advertising the initiative were posted to all eligible children. Paper surveys were given to those who participated, and phone surveys were conducted for those who didn't. Results were tabulated.

Results Runners (R) $n=16$. Non-Runners (NR) $n=14$. The mean age (9.4 years) and HbA1c (R: $8.061 \%$ or $64.6 \mathrm{mmol} /$ mol, NR: $8.077 \%$ or $64.8 \mathrm{mmol} / \mathrm{mol}$ ) levels for both groups were similar. Majority of both groups used continuous subcutaneous insulin therapy (R: 62.5\%, NR: 64.3\%). Interestingly, although R's had experienced a greater number of hypoglycaemic episodes in the previous two weeks (Mean R: $6.875,0-20$. NR: $5.43,2-10), 25 \%$ of R's reported the fear of hypoglycaemia would stop them from exercising, compared with $35.71 \%$ of NR's. This could be explained by the fact that NR's had experienced more severe hypoglycaemic episodes in the previous year (R: $1, \mathrm{NR}: 3$ ). Blood glucose monitoring by finger prick was more frequent amongst the R's with majority testing $10+$ times per day. $71.43 \%$ of NR's and only $43.75 \%$ of R's reported having zero friends with T1DM. $100 \%$ of R's had taken part in a group fun-run before whereas $92.86 \%$ of NR's had not.

Conclusion Demographics and relative diabetes control is similar across groups. Our results highlight differences in the attitudes and actions around hypoglycaemia and exercise and also in the support networks available to these children. The results from our survey will allow us to continue this initiative annually and future surveys will allow us to further study motivations and barriers to exercise in the paediatric population with T1DM.

\section{GP138 RESILIENCE ENHANCE THE PROTECTIVE IMPACT OF FAMILY FUNCTIONING ON DIABETES DISTRESS IN YOUTH WITH TYPE 1 DIABETES}

${ }^{1}$ Dan Luo*, ${ }^{2}$ Jingjing Xu, ${ }^{2}$ Wei He, ${ }^{2}$ Tao Yang, 'Mingzi Li. 'School of nursing, Peking University, Beijing, China; ${ }^{2}$ Department of Endocrinology, the First Affiliated Hospital of Nanjing Medical University, Nanjing, China

10.1136/archdischild-2019-epa.202

Objectives a) to explore the effect of family functioning on diabetes distress among adolescents with type 1 diabetes(T1D); and b) to examine whether resilience mediates the relationship between family functioning and diabetes distress.

Methods Youth with T1D recruited from a national endocrine center of a public hospital in China from May 2017 to October 2018. A total of 189 participants (aged 8-24 years) completed the survey about their resilience, family adaptability and cohesion, diabetes distress and provided demographic and clinical information. The moderation analyse was preformed to determine whether the resilience strengthens the protective impact of family adaptability and cohesion on diabetes distress. The simple slopes analyse was used to probe significant interactions.

Results The mean score of diabetes distress was $29.58 \pm 22.09$ with $31.7 \%$ of patients having severe diabetes distress. Multivariate linear regression analyses indicated that resilience enhanced the association that high family functioning had with low diabetes distress $(\beta=-0.22, t=-0.318, P=0.002)$. However, simple slopes found that benefits of high resilience for lower diabetes distress was only apparent in the context of low family adaptability and cohesion $(\beta=-0.941, t=-4.090, P=0.001)$.

Conclusions Many youth with type 1 diabetes reported severe diabetes distress which was associated with poor glycemic control and decreased quality of life. The finding of study suggest that family-based interventions which considered resilience factors are promising for youth with high diabetes distress especially for those have poor family functioning.

\section{GP139 THE INCIDENCE OF TRANSIENT PSEUDOHYPOALDOSTERONISM IN INFANCY IN IRELAND: A PROSPECTIVE WHOLE ISLAND SURVEILLANCE STUDY}

${ }^{1}$ Abhidhamma Kaninde* ${ }^{2}$ Mariana Grace, ${ }^{3}$ Caroline Joyce, ${ }^{4}$ Norman $F$ Taylor, Michael Riordan ${ }^{5},{ }^{6,7}$ Michael J O'Grady, ${ }^{8}$ Susan M O'Connell. ${ }^{1}$ Dept. of Paediatric and Child Health.Cork University Hospital, Cork, Ireland; ${ }^{2}$ Dept. of Paediatric and Child Health, Cork University Hospital, Cork, Ireland; ${ }^{3}$ Dept. of Clinical Biochemistry, Cork University Hospital, Cork, Ireland; ${ }^{4}$ Dept. of Clinical Biochemistry, Kings College London, London, UK ${ }^{5}$ Dept.Paediatric Nephrology, Our Lady's Children's Hospital, Crumlin, Dublin, Ireland; ${ }^{6}$ Dept. of Paediatrics, regional Hospital Mullingar, Mullingar, Ireland; 'Women's and Children's Health, University College Dublin, Dublin, Ireland; ${ }^{8}$ Dept of Paediatric and Child Health, Cork University Hospital, Cork, Ireland

\subsection{6/archdischild-2019-epa.203}

Aim To review the clinical features, presentation, investigations undertaken, and outcome of infantile salt-wasting presenting in the setting of urinary tract infection (UTI) and/or urinary tract malformation (UTM) over a two-year surveillance period on the island of Ireland. To estimate a population incidence based on the results and to make recommendations on the approach to management of this condition.

Methods A two-year (2013-14) prospective surveillance undertaken for the island of Ireland via the Irish and Ulster Paediatric Surveillance Units. Monthly-prepaid postcards were circulated to Consultant Paediatricians $(n=260)$ at all Paediatric Units on the island of Ireland.

Infants under one year of age presenting for the first time with hyponatremia (serum sodium $<130 \mathrm{mmol} / \mathrm{L}$ ) and/or hyperkalemia (serum potassium $>5.0 \mathrm{mmol} / \mathrm{L}$ ) associated with urosepsis/UTM were included.

Results Seven patients (six male), all aged younger than five months ( 3 weeks to 20 weeks) were reported during the study period. All had culture-proven UTI and five (71\%) also had an underlying UTM (one diagnosed antenatally). Four (57\%) patients had a documented elevated serum aldosterone supporting secondary pseudohypoaldosteronism (PHA) as the underlying diagnosis. Data on aldosterone was not reported in the other three patients but clinical features were suggestive of secondary PHA. All had an excellent outcome with full resolution of the electrolyte disturbance. No cases of primary PHA were submitted during the surveillance period. The estimated 